\title{
Regionalmanagement - Potenziale, Grenzen und Perspektiven
}

Ergebnisse einer Untersuchung in Sachsen-Anhalt im Rahmen einer Diplomarbeit (2003) im Fach Geographie an der FU Berlin

\section{Regional Management - Potentials, Limits and Perspectives}

\author{
Results of a study in Saxony-Anhalt in the framework of a diploma thesis (2003) \\ in the course "Geography" at the Free University of Berlin
}

\section{Kurzfassung}

Der Regionalmanagementansatz erfährt seit ungefähr einem Jahrzehnt eine zunehmende Aufmerksamkeit. Mit dem Ziel, die Leistungsfähigkeit dieses vielseits diskutierten Regionalmanagementansatzes zu untersuchen, wurde das Konzept aus theoretischer und praxisorientierter Perspektive in drei Regionen in Sachsen-Anhalt einer kritischen Analyse unterzogen. Die daraus gewonnenen Erkenntnisse werden in diesem Aufsatz dargelegt. Dabei werden u.a. die Regionalmanagementansätze der drei Beispielregionen vorgestellt und qualitätsbestimmende Faktoren identifiziert. Darüber hinaus werden Mängel bei der Implementierung aufgezeigt und daraus Empfehlungen für die zukünftige Ausgestaltung des Regionalmanagements abgeleitet.

\begin{abstract}
The concept of regional management has gained more and more importance over the last decade. Based on empirical studies of three regions in Saxony-Anhalt, the concept of regional management was closely examined from a theoretical and practical perspective to determine the efficiency of this concept. The results of this analysis are presented in this essay. The structures of the regional management of the three case studies are presented and quality criteria are identified. Problems of implementation are also indicated. On this basis, recommendations are drawn for the future implementation of regional management concepts.
\end{abstract}

Seit ungefähr zwei Jahrzehnten gewinnt die regionale Ebene in der räumlichen Planung, aber auch in wirtschaftlicher und politisch-administrativer Hinsicht an Bedeutung. Dies liegt u.a. an den veränderten politisch-administrativen Rahmenbedingungen wie z.B. der sinkenden staatlichen Steuerungskraft, dem gewandelten wirtschaftlichen Umfeld mit einem zunehmenden Standortwettbewerb im Zuge der Globalisierung, und es liegt auch an den veränderten finanziellen Rahmenbedingungen, die sich in Finanzengpässen öffentlicher Haushalte äußern (vgl. Danielzyk 1999).

In der Raumplanung führen diese Prozesse zu einem Wandel des traditionellen Planungsverständnisses, das beispielsweise von Fürst (2000) und Pohle (2003) ausführlicher behandelt wird. Dieser Wandel manifestiert sich u. a. in der Wahrnehmung der Region als neue Handlungs- und Umsetzungsebene. Darüber hinaus spiegelt er sich in der Erweiterung der traditionellen ordnungspolitischen Planungsinstrumente (Pflichtaufgaben) um neue entwicklungsorientierte Instrumente mit dem Ziel einer eigenständigen Steuerung der Regionalentwicklung (freiwillige Aufgaben). Vor diesem Hintergrund wird seit Mitte der 1990er Jahre in der planerischen Fachdebatte das Konzept des Regionalmanagements als neues regionales Kooperationsinstrument erörtert (vgl. Abb.1). Dieses soll die traditionelle Regionalplanung mittels eines ganzheitlichen querschnittsorientierten Ansatzes ergänzen, um den neuen Anforderungen gerecht zu werden. 
Zu gleicher Zeit ist auch ein Wandel in der Regionalpolitik zu beobachten, der u.a. bei Maier und Tödtling (2000) näher beschrieben wird. Dieser Wandel manifestiert sich in Regionalisierungsprozessen, d.h. in der Verlagerung und Bündelung von Kompetenzen auf der regionalen Ebene. Des Weiteren äußert er sich in der Ergänzung der klassischen Förderinstrumente um zwei neue Instrumente, die auf der Theorie der endogenen Regionalentwicklung beruhen. $\mathrm{Zu}$ diesen beiden neuen Förderinstrumenten gehört zum einen die Aufstellung regionaler Entwicklungskonzepte (REK). Zum anderen gehört dazu das Regionalmanagement, das durch die Mobilisierung regionaler Entwicklungspotenziale einen positiven Beitrag für die Regionalentwicklung leisten soll. Im August 2000 wurde das Regionalmanagement in den 29. Rahmenplan der Gemeinschaftsaufgabe für Regionale Wirtschaftsförderung (GRW), dem wichtigsten Förderinstrument der Regionalpolitik, aufgenommen. Seitdem können Regionen für die Durchführung eines Regionalmanagements eine auf drei Jahre ausgerichtete finanzielle Unterstützung beantragen. Derzeit werden Regionalmanagementansätze in ca. 40 Regionen innerhalb Deutschlands mit diesen Mitteln gefördert.

Vor dem Hintergrund des anhaltenden Bedeutungsgewinns des Regionalmanagementkonzepts stellt sich die Frage nach dessen Leistungsfähigkeit. Entsprechend ist Ziel dieses Beitrags, den theoretischen und methodischen Diskussions- und Erfahrungsstand zum Thema Regionalmanagement zusammenzufassen. Es gilt aufzuzeigen, was das Regionalmanagement in der Praxis leisten kann, wo die Grenzen des Konzepts be- stehen und was sich daraus für die künftige Ausgestaltung schließen lässt. Die im Folgenden dargestellten Erkenntnisse basieren vor allem auf der eingehenden Untersuchung der Regionalmanagements dreier Regionen in Sachsen-Anhalt, und zwar der Region Altmark, der Region Anhalt-Bitterfeld-Wittenberg und der Region Halle.

\section{Definition und Aufgabenfelder des Regionalmanagements}

Der Regionalmanagementansatz ist weder in der Fachliteratur noch im Rahmenplan der GRW eindeutig definiert, sodass in Abhängigkeit vom jeweiligen Regionen- und Planungsverständnis eine Vielzahl unterschiedlicher Definitionen und praktischer Ausprägungen existieren.

Regionalmanagement wird hier in Anlehnung an Fürst (1998 und 2001) verstanden als flexibles und situationsbezogenes Konzept zur querschnittsorientierten Steuerung, Führung und Gestaltung von Entwicklungsprozessen in einer Region. Die Implementation des Regionalmanagements erfolgt mittels regionaler Selbstorganisation und -steuerung mit dem Ziel der Förderung einer nachhaltigen Regionalentwicklung.

Auf der Grundlage der regionalpolitischen Strategie der endogenen Entwicklung ergänzt der ganzheitliche, integrative Regionalmanagementansatz die regionale Raumordnungspolitik auf handlungs- und prozessorientierte Weise (Knieling 2000, S. 8). Er bedient sich dabei der Prinzipien der strategischen Planung, der

Abbildung 1

Steuerungsdeterminanten der Regionalentwicklung

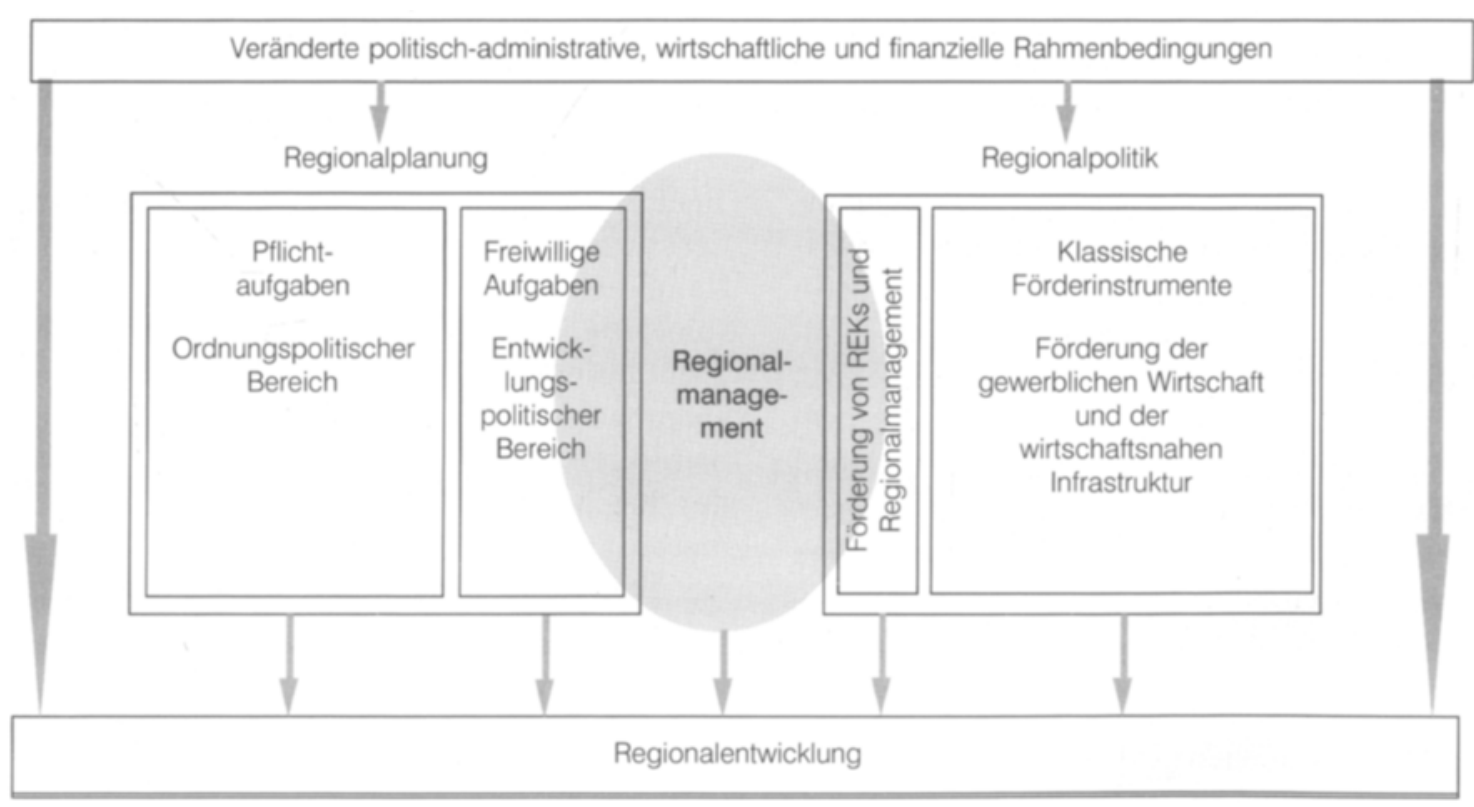


Kooperation und der Vernetzung. Der Anreiz zur freiwilligen Zusammenarbeit besteht in der gemeinsamen Problembetroffenheit der beteiligten Akteure. Auf der Basis von konsensualen regionalen Entwicklungsleitbildern und -konzepten steht die Planung und Umsetzung regionalbedeutsamer Projekte und Maßnahmen im Mittelpunkt, wobei durch den regionalen Blickwinkel Parallelplanungen vermieden und Synergieeffekte nutzbar gemacht werden sollen (vgl. Fürst 1998 und 2001 und Pohle 2003).

In Abgrenzung zu anderen querschnittsorientierten Kooperationsinstrumenten zeichnet das Regionalmanagement die Anlehnung an den wirtschaftswissenschaftlichen Managementbegriff aus, was u.a. von Troeger-Weiss (1998) und Fürst (1998) ausführlicher behandelt wird. Beim Regionalmanagement wird der Begriff der betrieblichen Steuerung auf die Steuerung von Entwicklungsprozessen in Regionen übertragen. Durch die Anwendung der klassischen Managementfunktionen und -phasen (Planung, Organisation, Personaleinsatz, Führung und Kontrolle) sollen Prozessabläufe effizienter gestaltet und brach liegende Ressourcen (wie z.B. regionale Selbsthilfepotenziale) in Wert gesetzt werden. Die Querschnittskoordination über den Managementansatz soll zudem als Bindeglied zwischen der Ordnungsplanung, der Umsetzungsebene (Fachressorts, Gemeinde) und der Privatwirtschaft wirken, um die derzeit bestehende Lücke zwischen diesen Bereichen zu schließen (Fürst 1998, S. 244). Durch koordiniertes Vorgehen und durch einen kostensparenden und effizient gestalteten Mitteleinsatz soll die optimale Nutzung der endogenen Faktoren gewährleistet werden.

Das Regionalmanagement ermöglicht darüber hinaus die Integration unterschiedlichster regionaler Akteure - in Abgrenzung beispielsweise zu Städtenetzen, bei denen ausschließlich die Kommunen miteinander kooperieren.

Aus den theoretisch und empirisch gewonnenen Erkenntnissen lässt sich eine Vielzahl an Aufgabenfeldern für das Regionalmanagement ableiten. Im Folgenden werden die Aufgabenbereiche in Anlehnung an Maier und Obermaier (2000, 77 ff.) und Troeger-Weiss (1998) aufgeführt, wobei es sich beim Kursivgedruckten um Tätigkeitsfelder handelt, die nach Meinung der Autorin in Zukunft einen höheren Stellenwert erhalten sollten:

\section{Aufgaben der Prozessgestaltung}

- Initiierung und Unterstützung regionaler Entwicklungsprozesse im Sinne eines Prozesspromotors (Schrittmacher)
- Steuerung (im Sinne des verstärkten Einsatzes der Managementfunktionen), Gestaltung, Organisation und Moderation regionaler Kommunikationsprozesse

- Mobilisierung von regionalen Selbsthilfepotenzialen

- Koordination und Vernetzung regionaler Projekte und Akteure

- Information und Beteiligung regionaler Akteure (u.a. durch eine intensive Öffentlichkeitsarbeit)

- Betreiben eines intensiven Motivationsmanagements zur Sicherstellung des langwährenden Bestehens der Zusammenarbeit (d.h. motivationssteigernde Maßnahmen wie z.B. eine transparente und strukturierte Steuerung des Prozesses und das Hervorheben des Kooperationsnutzens für die Beteiligten)

- Betreiben eines intensiven Relationshipmanagements zur Verbesserung der Akteursbeziehungen (einschl. der Vermittlung bei konfligierenden Interessen und in Konkurrenz- und Konfliktsituationen)

- Förderung eines intensiven Reflexionsmanagements im Sinne eines Controlling des Prozesses und der Ergebnisse u.a. durch den Einsatz von Kontrollinstrumenten, wie z.B. wissenschaftliche Begleitforschung und Evaluation

- Bereitstellung eines Beratungs-, Bildungs- und Informationsangebots, d.h. Beratung regionaler Akteure bzw. Projektträger als kostenpflichtige Dienstleistung

2. Inhaltliche Arbeit und thematische Ausrichtung

- Erarbeitung und Fortschreibung von Leitbildern und Entwicklungskonzepten unter breiter Beteiligung regionaler Akteure und unter Berücksichtigung der regionalen Entwicklungspotenziale und ihrer Grenzen

- Entwicklung und Initiierung von Modell- und Pilotprojekten (Innovationsmanagement)

- Stärkung der regionalen Identität bzw. des regionalen Gedankens durch eine intensive Öffentlichkeitsarbeit (Binnenmarketing)

- Förderung des Bekanntheitsgrades der Region bzw. Verbesserung des Images der Region durch ein intensives Regionalmarketing (Außenmarketing)

- Beteiligung an Wettbewerben im Bereich der Regionalentwicklung als Anreizmittel zur Zusammenarbeit

- Förderung eines intensiven Erfahrungsaustauschs zwischen den Regionen und zwischen Regionen und Ländern bzw. dem Bund, z. B. durch Diskussionsforen und themenbezogene Fachveranstaltungen 


\section{Regionalmanagement in Sachsen-Anhalt}

In Sachsen-Anhalt steht das Regionalmanagement in engem Zusammenhang mit der von der Landesregierung seit 1995 verfolgten regionalisierten Strukturpolitik. Die Regierung verfolgt damit einen kooperativen Entwicklungsansatz mit dem Ziel, die Mobilisierung regionaler Eigenkräfte und eine Auseinandersetzung mit den Entwicklungszielen in den einzelnen Regionen zu fördern. Im Zuge des Regionalisierungsprozesses konstituierten sich Ende der 1990er Jahre auf Anregung der Landesregierung die kommunalen Gebietskörperschaften zu fünf Regionen, wobei vier der fünf Regionen seit 2001 bzw. 2002 die Fördermöglichkeit zur Implementation eines Regionalmanagements in ihren Regionen wahrnehmen. Neben der Aufstellung von so genannten Regionalen Entwicklungskonzepten und der Implementierung des Regionalmanagements ist die Umstrukturierung der Regionalplanung in ein kommunalisiertes Modell ein weiteres Ergebnis der regionalisierten Strukturpolitik (Maier und Obermaier 2000, S. 34). Für diesen Zweck wurden in jeder der fünf gebildeten Planungsregionen Regionale Planungsgemeinschaften gegründet. Die Planungsregionen sind dabei identisch mit den Regionalmanagementregionen (Steinhart/Krome/Strähle 2003).

Zum 1. Januar 2004 wurden darüber hinaus die bis dahin bestandenen Regierungspräsidien aufgelöst und durch ein Landesverwaltungsamt als zentrale Bündelungs- und Koordinierungsbehörde ersetzt. Diese Umstrukturierung der Verwaltungsstruktur wirkt sich auch auf die Regionalmanagements der Regionen SachsenAnhalts aus, die gezwungen sind, sich an die neue Situation anzupassen.

Die Regionalmanagements der untersuchten drei Regionen sind durch unterschiedliche Ausprägungen charakterisiert, was ausführlich bei Schäffer (2003) diskutiert wird.

Bei der Wahl der Aufgabenfelder ist bei allen drei Regionen eine Dominanz wirtschaftlicher Themen zu beobachten. Als Schwerpunktaktivitäten sind dabei vor allem ein umfassendes Binnen- und Außenmarketing, der Ausbau und die Vernetzung touristischer Infrastrukturen sowie beschäftigungsschaffende Maßnahmen zu nennen. Hinsichtlich der Ausgestaltung des Regionalmanagements wurden von den Regionen zwei unterschiedliche Wege eingeschlagen: In der Region Altmark und in der Region Halle wird das Regionalmanagement ausschließlich als Koordinator bestehender Initiativen und Programme wie z.B. den Regionalen Entwicklungskonzepten und im Falle der Altmark auch des Regionalen Aktionsprogramms genutzt. Dagegen kommt dem Regionalmanagement in der Region Anhalt-Bitterfeld-Wittenberg die Funktion als Koordinator und vor allem Initiator ausgewählter Regionalmanagementprojekte, die in übergeordnete Entwicklungsleitlinien eingebettet sind, $\mathrm{zu}$. Als besonders positiv hat sich dabei eine flexible Ausgestaltung des Regionalmanagements erwiesen, d.h. dass auch im Verlauf der Kooperation neue Themenfelder in das Regionalmanagement aufgenommen werden, wenn veränderte äußere Rahmenbedingungen dies erfordern.

Die Akteursstruktur wird in allen drei Regionen von den lokalen Gebietskörperschaften dominiert. Die Einbeziehung von Akteuren aus dem wirtschaftlichen oder sozialen Sektor erfolgt nur in begrenztem Maße. Prozessbehindernd wirken die in allen drei Regionen ausgeprägten Konkurrenzbeziehungen zwischen den einzelnen Landkreisen, aber auch zwischen den einzelnen lokalen und - wenn wie in der Region Anhalt-Bitterfeld-Wittenberg vorhanden - regionalen Wirtschaftsförderungen. Besonders stark sind diese Konkurrenzsituationen in der Region Halle vorhanden. Dies kann u.a. darauf zurückgeführt werden, dass ein gemeinsames regionales Bewusstsein fehlt, da die Region keinen homogenen Natur- und Kulturraum umfasst. Ferner geht es der Region - im Gegensatz z. B. zur Altmark - in wirtschaftlicher Hinsicht „zu gut“, als dass daraus ein ausreichender Handlungsdruck resultieren würde, der die Akteure trotz der ausgeprägten Konkurrenzen zur intensiven Kooperation motivieren würde. In der Region Altmark gestaltet sich die Situation dagegen besser, da in der Altmark, als homogener Naturund Kulturraum, ein regionales Bewusstsein vorhanden ist. Des Weiteren besteht aufgrund der desolaten wirtschaftlichen Lage ein enormer Handlungsdruck, der sich positiv auf die Motivation der Akteure zur Kooperation auswirkt.

Aus organisatorisch-institutioneller Perspektive verfügen die Regionalmanagements aller drei Regionen über eine Grundausstattung an Kooperationsgremien: ein großes Versammlungsgremium mit Informationsfunktion, ein Steuerungsgremium mit Beschlussfunktion z.B. in Form einer Lenkungsgruppe, eine Geschäftsstelle mit Koordinierungsfunktion und ein Gremium mit Beratungsfunktion (z.B. in Form eines Projektbeirats); dazu ein oder mehrere Arbeitsgremien, die je nach Bedarf gebildet werden und die für die inhaltliche Arbeit zuständig sind.

In der Altmark ist das Regionalmanagement dabei lose an die formellen Strukturen der Regionalplanung angebunden, die neben der Wahrnehmung ihrer Pflichtaufgaben als Subauftragnehmer für informelle Planungen, wie z.B. das Regionalmanagement und das Städtenetz Altmark, fungiert (vgl. Abb. 2). 


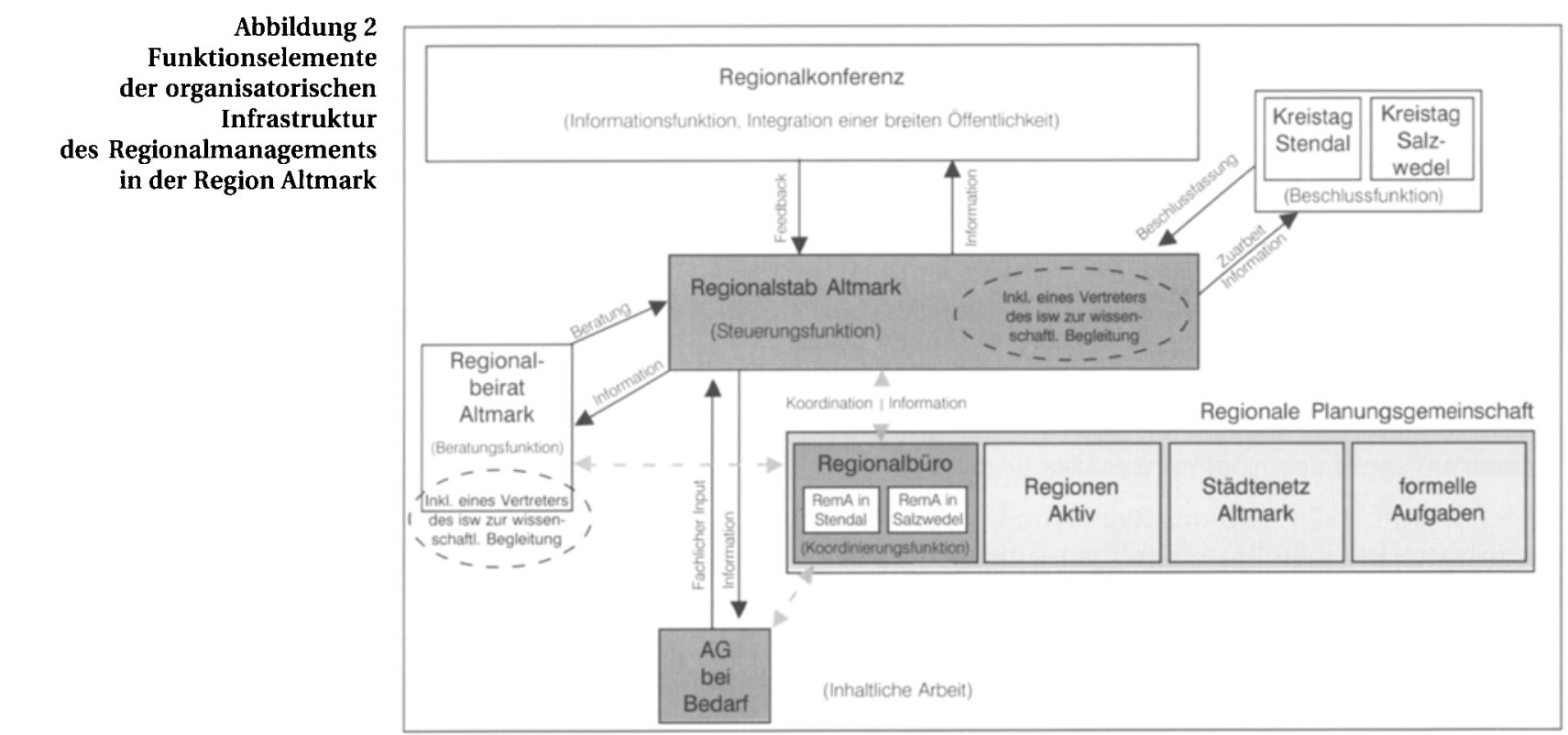

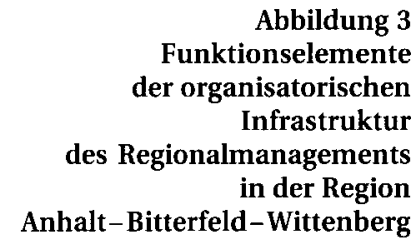

Abbildung 4 Funktionselemente der organisatorischen

Infrastruktur des Regionalmanagements in der Region Halle
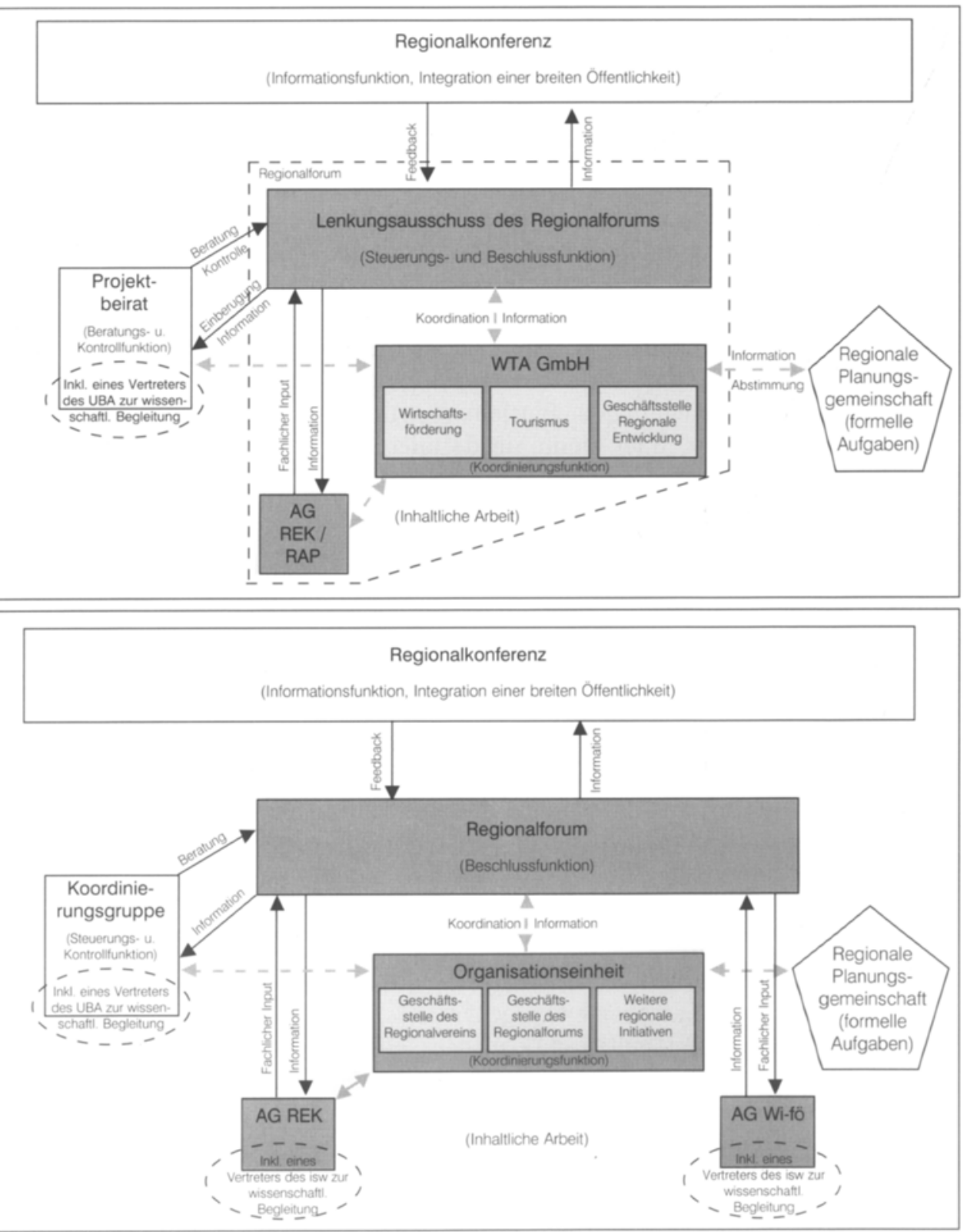
Im Regionalmanagement in der Region Anhalt-Bitterfeld-Wittenberg fungiert die für die gesamte Region zuständige Wirtschaftsförderung \& Tourismus ANHALT GmbH als Träger für das Regionalmanagement. Die hier verfolgte Verknüpfung der Aufgaben der regionalen Wirtschaftsförderung, des Tourismus und des Regionalmanagements in einer privatrechtlichen Institutionalisierungsform einer $\mathrm{GmbH}$ hat Modellcharakter (vgl. Abb. 3). Im Gegensatz zur Altmark kann beim Regionalmanagement in der Region Anhalt-Bitterfeld-Wittenberg von einem relativ hohen Institutionalisierungsgrad gesprochen werden.

In der Region Halle wird das Regionalmanagement in der privatrechtlichen Form eines gemeinnützigen Vereins durchgeführt (vgl. Abb. 4). Hinsichtlich des Institutionalisierungsgrades ist das Regionalmanagement zwischen der sehr schwach institutionalisierten Variante der Region Altmark und der stark institutionalisierten Form in Anhalt-Bitterfeld-Wittenberg einzuordnen.

Wie aus den Abbildungen 2 bis 4 hervorgeht, verfügen alle drei Regionen über eine gut ausgebaute organisatorische Infrastruktur, die die Durchführung der Regionalmanagements in Zusammenarbeit mit regionalen Akteuren ermöglicht. Dennoch bestehen hinsichtlich der Nutzung dieser Infrastruktur Konfliktbereiche, die die Qualität des Regionalmanagements abschwächen. So ist z.B. in allen drei Regionen die Aufgabenverteilung zwischen den einzelnen Regionalmanagementgremien bzw. zwischen den Regionalmanagementgremien und weiteren regionalen Einrichtungen nur unzureichend definiert, oder wenn sie definiert ist, wird sie nur unzureichend umgesetzt. In diesem $\mathrm{Zu}$ sammenhang seien insbesondere die lokalen Wirtschaftsförderungen genannt, die eher in Konkurrenz zum Regionalmanagement stehen, als dass sie sinnvollerweise ihre Politiken und Maßnahmen aufeinander abstimmen. Auch die Zusammenarbeit und Abstimmung zwischen der formellen Regionalplanung und dem informellen Regionalmanagement in der Region Halle und in der Region Anhalt-Bitterfeld-Wittenberg ist als ausbaufähig zu bezeichnen. Langwierige Entscheidungsabläufe z. B. bei Projektgenehmigungen innerhalb des Regionalmanagements stellen zusätzlich in allen drei Regionen ein großes Hindernis dar.

Hinsichtlich des Institutionalisierungsaspekts ist es auffallend, dass alle drei Varianten trotz ihrer unterschiedlichen Institutionalisierungsform durch Engagement- und Akzeptanzprobleme gekennzeichnet sind, wenn auch unterschiedlich stark ausgeprägt und auf verschiedenen Ursachen beruhend, wie z.B. der fehlenden regionalen Identität in der Region Halle und in der Region Anhalt-Bitterfeld-Wittenberg, und aufgrund unzureichender Institutionalisierung in der Region Altmark.

\section{Regionalmanagement zwischen Theorie und Praxis}

Im Rahmen der Untersuchung der drei Fallbeispiele wurden Diskrepanzen zwischen dem in der Fachliteratur definierten Konzept des Regionalmanagements und dessen praktischer Ausgestaltung festgestellt. Diese resultieren

1. in erster Line aus der Abhängigkeit der Regionen von Fördermitteln* und

2. zum geringeren Maße aus den Differenzen zwischen den unterschiedlichen Logiken bzw. Funktionsweisen betriebswirtschaftlich geführter Unternehmen und der auf Freiwilligkeit beruhenden regionalen Zusammenschlüsse.

Dies soll an drei Beispielen verdeutlicht werden:

Bei einer streng genommenen Betrachtung wird das in der Theorie hervorgehobene Ziel der Förderung einer nachhaltigen Regionalentwicklung aufgrund der Dominanz wirtschaftlicher Aufgabenfelder bei gleichzeitiger Vernachlässigung sozialer und ökologischer Aspekte oft nur in begrenztem Maße erreicht. Es ist nur im engeren Sinne insofern gewährleistet, als dass eine verbesserte Koordinierung zu einem sparsameren und effektiveren Ressourcenumgang führt. In diesem Bereich besteht jedoch noch umfassender Verbesserungsbedarf, insbesondere in der Koordination und Vernetzung regionaler Aktivitäten und Akteure.

Ein weiterer Gegensatz besteht im Hinblick auf die regionale Selbststeuerung. Diese hat auf der einen Seite durch die Implementation des Regionalmanagements eine Aufwertung erfahren und findet in den Regionen eine breite Anwendung. Auf der anderen Seite ist zu beobachten, dass sie in starkem Maße dem externen Einfluss der Bindung an die Förderprogramme unterworfen ist, was die Handlungsspielräume der Regionen stark einschränkt. Das Konfliktfeld besteht darin, dass die Organisation der regionalen Selbststeuerung den Fördermittelgebern nur in begrenztem Umfang als Ergebnis vermittelbar ist, obwohl es ein entscheidendes Merkmal und auch ein Ergebnis des Regionalmanagements darstellt.

Auch im Bereich der Umsetzung des klassischen Managementkonzepts als besonderes Merkmal des Regionalmanagements bestehen Differenzen zwischen Theorie und Praxis. Die Praxis zeigt, dass das betriebswirtschaftliche Managementkonzept nicht direkt bzw. unreflektiert in den Kooperationskontext übertragen werden kann. Aus diesem Grund ist die Kritik von Sedlacek (2003) am Regionalmanagementbegriff sicher gerechtfertigt. 
Es stellt sich nun die Frage, ob die beschriebenen Divergenzen verringert werden können. Im Bereich der Erfüllung des Ziels einer nachhaltigen Regionalentwicklung ist dies mit großer Wahrscheinlichkeit nicht bzw. nur sehr begrenzt möglich. Da das Prinzip der Freiwilligkeit im Regionalmanagement eine zentrale Größe darstellt, werden die als unattraktiv wahrgenommenen sozialen und ökologischen Aufgabenfelder auch in Zukunft selbst bei einem intensiven Motivationsmanagement nur einen geringen Anklang finden.

Der Abhängigkeit von den Fördermitteln kann dagegen durch den Aufbau selbsttragender Strukturen entgegengewirkt werden. Und im Bereich der Übertragung der klassischen Managementlehre auf das Regionalmanagement gilt es zu klären, welche der klassischen Managementfunktionen für die Qualität und Langlebigkeit eines Regionalmanagements von besonderer Bedeutung sind und auf welche Weise sie besser an die besondere Situation des Regionalmanagements angepasst und besser eingesetzt werden können.

\section{Erfolgsfaktoren und Grenzen}

An den drei Fallbeispielen hat sich gezeigt, dass die Qualität und die Verstetigung des Regionalmanagements nicht in erster Linie von der Organisationsstruktur bzw. der Institutionalisierungsform, sondern von der Willensbekundung der regionalen Akteure abhängig sind. Sind die regionalen Akteure nicht bereit, die zeitlichen und finanziellen Ressourcen für eine solche Kooperation aufzubringen, kann auch mit Hilfe der innovativsten und effektivsten Organisations- bzw. Institutionalisierungsform kein Regionalmanagement durchführt werden. Neben diesem unerlässlichen regionalen Willen ist als zweiter wesentlicher Faktor die ausreichende Finanzausstattung zu nennen. Auch die Art der Institutionalisierungsform, die Organisationsstruktur und die politischen und administrativen Rahmenbedingungen spielen für die Qualität des Regionalmanagements eine Rolle. Diese Faktoren sind jedoch im Vergleich zur regionalen Akzeptanz und der Finanzausstattung durch eine untergeordnete Bedeutung gekennzeichnet.

Bedingt durch regionsinterne und -externe Faktoren sieht sich das Regionalmanagement mit natürlichen und systemimmanenten Grenzen konfrontiert, die es in seiner Leistungsfähigkeit und Wirksamkeit einschränken.
Die Grenzen des Regionalmanagements bestehen vor allem darin, dass

- das Regionalmanagement aufgrund des Prinzips der Freiwilligkeit nur konsensuale Themenfelder bearbeiten kann, d.h. Themenfelder, in denen von allen Beteiligten ein konkreter Handlungsbedarf gesehen wird und für die die notwendige Motivation vorhanden ist

- auf der regionalen Ebene keine legitimierte Institution, z. B. in Form eines Regionalparlaments, existiert, der umfangreiche Kompetenzen insbesondere zur Ressourcenbündelung und -verteilung obliegen.

- dem Regionalmanagement keine umfassenden Kompetenzen hinsichtlich größerer entwicklungsbestimmender Entscheidungen, wie z. B. Investitionsentscheidungen, obliegen. Das Regionalmanagement kann dementsprechend nur punktuelle Schwächen angehen, nicht aber größere Themen wie die Abwanderungsproblematik und die geringe Investitionsfreudigkeit von Unternehmen. Es kann lediglich Empfehlungen für große entwicklungsrelevante Entscheidungen geben.

- im Regionalmanagement Akteure unterschiedlichster Gesellschaftsformen aufeinandertreffen, die sich nicht gerne in die „Karten“ schauen lassen wollen. Die Stärke des Regionalmanagements (Integration unterschiedlichster Akteure) stellt somit gleichzeitig auch eine Einschränkung dar, die besondere Anforderungen an das Management stellt.

- dem Regionalmanagement in seiner jetzigen Ausgestaltung nur ein begrenzter Umfang an personellen und finanziellen Ressourcen zur Verfügung stehen, was die Handlungsspielräume eingrenzt. Demgegenüber ist das Regionalmanagement mit einem hohen Erwartungsdruck konfrontiert, der vor dem begrenzten Mittelumfang aber zu enttäuschenden Ergebnissen führen kann.

- das Regionalmanagement in seiner jetzigen Ausgestaltung in starkem Maße durch die Abhängigkeit von Fördermitteln gekennzeichnet ist, was das Erzielen messbarer Ergebnisse gegenüber der eigentlichen Prozessgestaltung in den Vordergrund rückt.

- das Regionalmanagement in seiner jetzigen Ausgestaltung als zeitlich beschränkter Prozess angelegt ist, wobei der zur Verfügung stehende Zeitraum zum Erzielen größerer Ergebnisse nicht ausreicht.

- die beteiligten Akteure oft in einem Konkurrenzverhältnis zueinander stehen. Besonders hervorzuheben sind dabei die Konkurrenzen zwischen den einzelnen Landkreisen, zwischen den lokalen und regionalen Wirtschaftsförderungen sowie zwischen der Region per se und der Landesebene. 


\section{Perspektiven und Empfehlungen}

\subsection{Perspektiven}

Die Perspektiven des Regionalmanagements werden

1. vor allem von der Willensbekundung der regionalen Akteure und

2. vom Umfang der Finanzkraft, die von den Regionen getragen werden kann,

abhängig sein.

In Regionen, in denen der ausreichende Wille fehlt, wird das Regionalmanagement mit großer Wahrscheinlichkeit nach Auslaufen der derzeitigen Förderperiode nicht fortgeführt werden. Nur in Regionen, in denen wirklich die Notwendigkeit in der Fortsetzung gesehen wird und die es geschafft haben, in der derzeitigen Förderperiode relativ stabile Kooperationsstrukturen aufzubauen, wird eine Fortführung der Kooperation über das Regionalmanagement möglich sein. Stabile Kooperationsstrukturen bedeuten in diesem Zusammenhang, dass sie sowohl den regionsinternen Interessensdivergenzen als auch den Konkurrenzverhältnissen standhalten können und dass die Region sich um eigenständige, innovative Finanzierungsmöglichkeiten bemüht hat. Auf die Nutzung alternativer Finanzierungsformen gehen u.a. Maier und Obermaier (2000) näher ein. In diesem Feld besteht noch weiterer Forschungsbedarf, insbesondere im Hinblick auf die Art geeigneter Finanzierungsarten und den konkreten Beitrag, den diese leisten können.

\subsection{Empfehlungen}

Die im Folgenden aufgeführten Empfehlungen wurden als Ergebnis der empirischen Untersuchung der drei Beispielregionen identifiziert. Sie richten sich zum einen an die in das Regionalmanagement involvierten Akteure der regionalen Ebene und zum anderen an die externen Experten mit Consulting- oder Coachingfunktion für den Regionalmanagementprozess. Es sei an dieser Stelle angemerkt, dass sie natürlich nicht auf alle zur Zeit in Deutschland durchgeführten Regionalmanagementansätze übertragen werden können. Sie gelten vielmehr als Anregungen und müssen im jeweiligen regionalen Kontext auf ihre Übertragbarkeit geprüft werden.

\section{Empfehlungen an die regionale Ebene}

Aufgabenfelder

- Grundsätzlich sollte dem Regionalmanagement eine strategische Planung zugrunde liegen. Aus den Fallstudien hat sich ergeben, dass es sinnvoll ist, sich auf der Basis von gesetzten und konsequent verfolgten Prioritäten auf eine begrenzte Anzahl von Themen zu konzentrieren und diese intensiv zu bearbeiten anstelle sich eine Vielzahl von Aufgabenfeldern vorzunehmen und diese nur oberflächlich anzugehen. Ferner hat sich gezeigt, dass die Aufgabenfelder zu Beginn einer Förderperiode anhand der gesetzten Prioritäten festgelegt werden müssen. Im weiteren Verlauf aber sollte das Regionalmanagement so offen angelegt sein, dass in Ausnahmefällen auch neue Themen aufgenommen werden können, wenn dies veränderte Rahmenbedingungen verlangen. So kann es z.B. sinnvoll sein, bei einer sich konsequent verschlechternden Wirtschaftsentwicklung mit zunehmenden Arbeitslosenzahlen einen stärkeren Schwerpunkt auf beschäftigungsschaffende Maßnahmen zu legen.

- Im Bereich der Prozessgestaltung hat sich in den Beispielregionen gezeigt, dass insbesondere folgende strategische Managementbereiche einen höheren Stellenwert erhalten sollten:

Das Motivationsmanagement, um die regionale Akzeptanz zu verbessern.

Das Relationshipmanagement, um den ausgeprägten Konkurrenzverhältnissen zwischen den regionalen Akteuren entgegenzuwirken.

Das Reflexionsmanagement, um einen größeren Nutzen aus gemachten Erfahrungen zu ziehen. Das beinhaltet auch, dass Fehler nicht negativ gewertet, sondern als Potenzial angesehen werden. Eine wissenschaftliche Begleitung durch externe Fachexperten kann hier einen unterstützenden Beitrag leisten, indem durch die Förderung einer kritischen Reflexion des Regionalmanagementprozesses neue Impulse bewirkt werden.

- Generell können nicht alle auf dem betriebswirtschaftlichen Managementkonzept beruhenden $\mathrm{Ma}$ nagementfunktionen im Maßstab eins zu eins in das Regionalmanagementkonzept übertragen werden. Die Wahrnehmung von Steuerungsaufgaben (Koordinierungsfunktion, klare transparente Aufgabenverteilung) sollte in Zukunft jedoch intensiver verfolgt werden.

- Der Informations- und Erfahrungsaustausch zwischen den regionalmanagement-implementierenden Regionen, v.a. zwischen den einzelnen Regionalmanagern, sollte intensiviert werden, um gegenseitige Lernprozesse $\mathrm{zu}$ fördern und um gemeinsame Interessen gegenüber den übergeordneten Ebenen (z.B. Ministerien) vertreten zu können. 


\section{Akteursstruktur}

- Grundsätzlich hat sich in den Regionen gezeigt, dass eine stärkere Integration von Akteuren außerhalb von Verwaltung und Politik intensiver verfolgt werden sollte. Auf diese Weise wird man der Querschnittsorientierung des Regionalmanagements auch innerhalb der Akteursstruktur gerecht. Ferner könnte damit sichergestellt werden, dass eine breitere Öffentlichkeit erreicht wird, was wiederum die regionale Akzeptanz und die Leistungsfähigkeit des Regionalmanagements erhöhen würde. Die Entwicklung von neuen Beteiligungsformen ist gegebenenfalls zu unterstützen. Das Regionalmanagement könnte in diesem Kontext stärker die Funktion eines Vermittlers übernehmen und die Integration ungenügend einbezogener Institutionen fördern.

\section{Organisatorische Infrastruktur}

- Die personelle Ausstattung der Geschäftsstelle sollte mindestens zwei Mitarbeiter umfassen, um die nötigen Aufgaben angemessen erfüllen zu können. Ist diese Ausstattung, die für die Mehrzahl der Regionen eine Personalaufstockung bedeuten würde, nicht zu gewährleisten, sollte eine Reduzierung der gestellten Aufgaben bzw. eine noch stärkere Prioritätensetzung erfolgen.

- In den drei Fallstudien fiel auf, dass eine unzureichende Definition, Darstellung und Umsetzung einer klaren und transparenten Aufgabenverteilung prozessbehindernd wirkt. Vor diesem Hintergrund sollte eine klare Aufgabenverteilung zwischen den Regionalmanagementgremien, aber auch zwischen dem Regionalmanagement und bestehenden Einrichtungen, wie z. B. den lokalen Wirtschaftsförderungen, festgelegt und umgesetzt werden. Auf diese Weise könnte eine höhere Effektivität der Prozesse erreicht werden und konkurrierende Einrichtungen könnten von dem Nutzen des Regionalmanagements leichter überzeugt werden, da zeit- und ressourcenaufwendige Parallelplanungen reduziert werden würden.

- Ferner hat die Untersuchung ergeben, dass die Zusammenarbeit und Abstimmung zwischen den Regionalmanagementgremien und den regionalen Institutionen intensiviert und in ihrer Qualität verbessert werden sollte. Darunter fallen beispielsweise die Beziehungen zwischen dem Regionalmanagement und der Regionalplanung.

- Empfehlenswert wäre auch, dass die Genehmigungsverfahrensabläufe und die Entscheidungsprozeduren für die im Regionalmanagement anvisier- ten Projekte verkürzt werden. Um ein besseres Kooperations- aber auch Investitionsklima zu schaffen, ist schnelleres Handeln eine wichtige Voraussetzung.

\section{Institutionalisierung}

- An den drei Fallstudien hat sich gezeigt, dass die Institutionalisierung einen positiven Einfluss auf die regionalen Kooperationsbemühungen haben kann. Fragen der Verstetigung sollten dementsprechend frühzeitig diskutiert und entsprechende Maßnahmen rechtzeitig umgesetzt werden. Um die Kooperationsstruktur nicht unnötig zu verkomplizieren, sollte die Institutionalisierung dabei die bestehende Institutionenlandschaft berücksichtigen und -wenn dies sinnvoll erscheint- bestehende Kooperationsstrukturen nutzen.

- Die Institutionalisierung per se darf aber nicht als eine einmalige Aktion verstanden werden. In der Regel ist die kontinuierliche Durchführung von $a k$ zeptanzverbessernden Maßnahmen, wie z. B. eine intensive Öffentlichkeitsarbeit oder die Einführung transparenter Strukturen, notwendig, um eine langfristige regionale Akzeptanz gewährleisten zu können.

\section{Finanzierung}

- Vor dem Hintergrund der derzeit schwierigen kommunalen Haushaltslage und den damit verbundenen Grenzen der kommunalen finanziellen Förderung solcher Kooperationsstrukturen, sollten sich die Regionen stärker für den Aufbau selbsttragender Strukturen einsetzen. Auf diese Weise könnte die Abhängigkeit von externen Förderprogrammen reduziert werden. Dies wiederum würde die Flexibilität und die Handlungsspielräume des Regionalmanagements erhöhen, da dann die Erfüllung von teilweise unangepassten Förderkriterien nicht mehr im Mittelpunkt der Kooperationsbemühungen stehen würde. Auch sollte die Erforschung neuer innovativer Finanzierungsformen vor diesem Hintergrund unterstützt werden.

- Bei den Fallstudien wurde deutlich, dass eine Aufteilung der Mittel auf einzelne Teilräume der Bildung eines regionalen Verständnisses entgegenwirkt und die Initiierung isolierter Einzelprojekte ohne regionalen Bezug fördert. Um dies zu verhindern, sollten die Regionalmanagementgelder zentral für die Gesamtregion und nicht dezentral von einzelnen Teilräumen verwaltet werden. 


\section{Empfehlungen an die Landes- und Bundesebene}

- Die Förderung von Regionalmanagementansätzen im Rahmen der GRW sollte auch nach Beendigung der derzeitigen Förderperiode fortgeführt werden. Vor dem Hintergrund, dass sich auch die Bundesebene einer angespannten Haushaltslage gegenübersieht, sollte sich die Förderung von Regionalmanagementansätzen auch in Zukunft auf die strukturschwächsten Regionen konzentrieren. Ohne entsprechende externe Anreize können solche Ansätze in den Regionen meist nicht durchgesetzt werden. Von vornherein ist aber ausdrücklich zu erwähnen, dass es sich - damit in den Regionen keine falschen Hoffnungen erzeugt und Nehmermentalitäten kreiert werden - nur um eine zeitlich begrenzte Förderung handelt. Die Betonung der zeitlichen Begrenzung bietet dabei auch einen Anreiz selbsttragende Strukturen frühzeitig aufzubauen. Über die Anschubfinanzierung hinaus sollte nur in besonders strukturschwachen Regionen die Möglichkeit bestehen, die erste Regionalmanagementförderperiode um weitere drei Jahre zu verlängern. Grundsätzlich sollten die finanzielle Förderungen in Zukunft stärker in Form einer degressiven Förderung erfolgen, um den Regionen einen kontinuierlichen anstelle eines abrupten Übergangs in die Eigenfinanzierung nach Auslaufen der Förderung zu ermöglichen. Für die besonders strukturschwachen Regionen würde das bedeuten, dass sie zwar eine Folgeförderung erhalten, jedoch auf einem niedrigeren Niveau.

- Darüber hinaus sollten insbesondere für strukturschwache Regionen finanzielle Förderungen zur Projektfinanzierung - unter der Voraussetzung der Erbringung des geforderten Eigenbetrags - bereitgestellt werden. Nur auf diese Weise kann in der derzeit angespannten kommunalen Haushaltslage die Gemeinnützigkeit des Regionalmanagements inklusive der Durchführung „unrentabler“ sozialer Projekte gewährleistet werden.

- Bei der Erarbeitung von Förderprogrammen sollten die regionalen Belange stärker berücksichtigt bzw. ausgewählte Regionenvertreter beteiligt werden. Um die Wirksamkeit des Regionalmanagements nicht zu behindern, sollte u.a. der Mitteleinsatz von den Regionen flexibler gestaltet werden dürfen. Auch sollte das Erreichen messbarer Ergebnisse einen geringeren Stellenwert erhalten. Und das Erzielen von Einnahmen seitens des Regionalmanagements sollte nicht mit Fördermittelstreichung bzw. -reduzierung bestraft werden, da sonst jeglicher Anreiz zur Erschließung alternativer Finanzierungsquellen genommen wird.
- Eine institutionelle Unterstützung in Form eines Patenschaftsmodells, bei dem hochrangige Personen wie z.B. Vertreter der Landesministerien die Funktion eines Sprachrohrs für die Region übernehmen, sollte den Regionen angeboten werden. Durch die Teilnahme solcher "Regionenvertreter" an bedeutenden regionalen Gremiensitzungen wird die Landesebene für die regionalen Belange sensibilisiert. Gleichzeitig wird damit den regionalen Akteuren motivationssteigernd wirkende Anerkennung für ihre Bemühungen entgegengebracht.

- Die Vielfalt der zum Großteil nicht aufeinander abgestimmten Förderprogramme sollte reduziert werden. Darüber hinaus sollte es den Regionen ermöglicht werden, Mittel aus Förderprogrammen mit ähnlicher Zielstellung gebündelt einsetzen zu dürfen.

- In Sachsen-Anhalt hat sich gezeigt, dass politische Unsicherheiten und inkonsequente politische Entscheidungen (z. B. im Zuge der Regionalisierung der Strukturpolitik) sich negativ auf das Regionalmanagement ausgewirkt haben. Es wäre daher wünschenswert, dass die Landesregierung den Regionen eine verlässliche, langfristige, klare politische Schwerpunktsetzung bietet, um die Ansätze der regionalen Selbstorganisation, die sich als Ergebnis langjähriger Bemühungen gebildet haben, nicht $\mathrm{zu}$ gefährden.

\section{Fazit}

In den vorangegangenen Kapiteln wurde das Konzept des Regionalmanagements in seiner Vielschichtigkeit aus praxisorientierter Sicht diskutiert. Ferner wurden Mängel bei der Implementierung und Grenzen in der Wirksamkeit des Regionalmanagements genannt. Daraus darf jedoch nicht gefolgert werden, dass das Regionalmanagement zwar in der Theorie ein durchaus vielversprechender Ansatz, in der Praxis aber - aufgrund der gegebenen Rahmenbedingungen nicht zu realisieren ist.

Schon die Einführung eines Regionalmanagements in einer Region per se kann als ein Qualitätsmerkmal und als eine besondere Leistung angesehen werden. Bietet es doch einen Rahmen, in dem regionale Akteure im Interesse des Gesamtwohls vernetzt zusammenarbeiten können. Dies nämlich erst ermöglicht die Bearbeitung von Themenfeldern, die aufgrund ihres sektorübergreifenden Charakters außerhalb der Zuständigkeitsbereiche einzelner regionaler Akteure liegen und aus diesem Grund ohne den Einsatz des Regionalmanagements gar nicht erst bearbeitet werden würden. Ferner kann dieses Konzept einen effizienten 
Einsatz der derzeit begrenzten Ressourcen fördern. Vor diesem Hintergrund ist die Fortführung dieses Ansatzes - unter Berücksichtigung seiner Potenziale und Grenzen und unter Anpassung an die jeweiligen regionalen Besonderheiten - in jedem Falle zu empfehlen. Ziel muss es sein, realistische Erwartungen an dieses Konzept zu stellen und es so an die restriktiven Rahmenbedingungen anzupassen, dass ein größtmöglicher Nutzen daraus gezogen werden kann.

\section{Anmerkung}

* In der Praxis wurde das Regionalmanagement im Rahmen der GRW als Förderinstrument eingeführt, um den sinnvollen Einsatz von Fördergeldern zu ermöglichen und die wirtschaftliche Entwicklung der Regionen zu fördern. So ist es zu verstehen, dass in der Praxis die Erfüllung von Förderkriterien im Mittelpunkt steht und dass das "eigentliche" Regionalmanagement darüber oft in den Hintergrund gedrängt wird.

\section{Literatur}

Danielzyk, Rainer (1999): Liegt die Zukunft in den Regionen? In: ARL (Hrsg.): Regionalentwicklung in Thüringen vor dem Hintergrund des Zusammenwachsens des Europas der Regionen. Planung, Praxis, Perspektiven. Arbeitsmaterialien 246, S. 54-63. - Hannover

Fürst, Dietrich (1998): Projekt- und Regionalmanagement. In: ARL (Hrsg.): Methoden und Instrumente der räumlichen Planung, S. 237-253. - Hannover

Fürst, Dietrich (2000): Wandel der Regionalplanung im Kontext des Wandels des Staates? In: Fürst, Dietrich; Müller, Bernhard (Hrsg.): Wandel der Planung im Wandel der Gesellschaft. IÖRSchriften. Bd. 33, S. 9-29. - Dresden

Fürst, Dietrich (2001): Regionalmanagement als Instrument einer nachhaltigen Raumentwicklung. In: Behrens, Hermann; Dehne, Peter; Kaether, Johann: Regionalmanagement - Der Weg zu einer nachhaltigen Regionalentwicklung. Referate im Rahmen des gleichnamigen Fachsymposiums am 19. Oktober 2000, S. 1-12. - Neubrandenburg
Knieling, Jörg (2000): Leitbildprozesse und Regionalmanagement. Ein Beitrag zur Weiterentwicklung des Instrumentariums der Raumordnungspolitik. - Frankfurt/Main

Maier, Jörg; Obermaier, Frank (2000): Regionalmanagement in der Praxis - Erfahrungen aus Deutschland und Europa. Chance für Bayern. - München

Maier, Gunther; Tödtling, Franz (2000): Regional- und Stadtökonomik 2. Regionalentwicklung und Regionalpolitik. Springers Kurzlehrbücher der Wirtschaftswissenschaften. - Wien

Pohle, Hans (2003): Regionalmanagement - Eine notwendige Ergänzung regionalplanerischer Aufgaben in Ostdeutschland. In: Schenkhoff, Hans Joachim (Hrsg.): Regionalmanagement in der Praxis - Beispiele aus Sachsen, Sachsen-Anhalt und Thüringen. Arbeitsmaterial Nr. 298 der ARL, S. 11-31. - Hannover

Schäffer, Verena (2003): Regionalmanagement in Sachsen-Anhalt. Theoretische Grundlagen und praktische Ausgestaltung im Vergleich dreier Regionen. Diplomarbeit im Fachbereich Geographie an der Freien Universität. METAR-Manuskripte zur empirischen, theoretischen und angewandten Regionalforschung der Freien Universität Berlin, Bd. 44. 2003. - Berlin

Sedlacek, Peter (2003): Regionalmanagement - ein Konzept zwischen Region und Organisation. In: Schenkhoff, Hans Joachim (Hrsg.): Regionalmanagement in der Praxis - Beispiele aus Sachsen, Sachsen-Anhalt und Thüringen. Arbeitsmaterial Nr. $298 \mathrm{der}$ ARL, S. 1-10. - Hannover

Steinhart, Anita; Krone, Frank; Strähle, Simone: Regionalmanagement - Ansätze in der Praxis - Ergebnisse einer empirischen Untersuchung. In: Schenkhoff, Hans Joachim (Hrsg.): Regionalmanagement in der Praxis - Beispiele aus Sachsen, Sachsen-Anhalt und Thüringen. Arbeitsmaterial Nr. 298 der ARL, S. 46-84. - Hannover

Troeger-Weiss, Gabi (1998): Regionalmanagement. Ein neues Instrument der Landes- und Regionalplanung. - Augsburg

Verena Schäffer

Elmshorner Straße 36

D - 14167 Berlin

E-Mail: vschaeffer@gmx.de 